International Journal of Wireless \& Mobile Networks (IJWMN) Vol. 6, No. 3, June 2014

\title{
Zigbee BASED WeARABle ReMote HealthCARE MONITORING SYSTEM FOR ELDERLY PATIENTS
}

\author{
Khalifa AlSharqi, Abdelrahim Abdelbari, Ali Abou-Elnour, and Mohammed \\ Tarique
}

Department of Electrical Engineering, Ajman University of Science and Technology, Fujairah, United Arab Emirates

\begin{abstract}
Remote health care monitoring system (RHCMS) has drawn considerable attentions for the last decade. As the aging population are increasing and at the same time the health care cost is skyrocketing there has been a need to monitor a patient from a remote location. Moreover, many people of the World are out of the reach of existing healthcare systems. To solve these problems many research and commercial versions of RHCMS have been proposed and implemented till now. In these systems the performance was the main issue in order to accurately measure, record, and analyze patients' data. With the ascent of wireless network RHCMS can be widely deployed to monitor the health condition of a patient inside and outside of the hospitals. In this work we present a ZigBee based wireless healthcare monitoring system that can provide real time online information about the health condition of a patient. The proposed system is able to send alarming messages to the healthcare professional about the patient's critical condition. In addition the proposed system can send reports to a patient monitoring system, which can be used by the healthcare professionals to make necessary medical advices from anywhere of the World at any time.
\end{abstract}

\section{KEYWORDS}

ZigBee, Wireless, patient, monitoring, healthcare, wearable, sensor, networks, data publishing

\section{INTRODUCTION}

Over the recent years remote health care monitoring systems for the elderly people have drawn considerable attentions. According to UNFPA, the global population is no longer young for the first time in the history [1]. Population ageing is affecting the entire world and is happening in all regions. But, it is progressing at a faster rate in the developing countries. Seven out of the fifteen countries in the developing world have more than 10 million old people. By the year 2050 another fifteen developing countries are expected to have 10 million old people. It is worthwhile to mention here that the average life expectancy in the United States was 47.3 years in 1900. But, it has increased to 68.2 years and 77.3 years in 1950 and 2002 respectively [2,3]. People are living longer because of better nutrition, sanitation, medical advances, education, economic well-being, and health care. Population ageing poses challenges to individuals, families, and societies. By adopting proper policies societies should be prepared for an ageing world. Overall, the older people should not be considered as a burden for the society. Their wisdom, energy, and experience are added advantages for us to take care of the challenges of the $21^{\text {st }}$ century. In order to keep the ageing population healthy we have to deal with some challenges. The major challenge for us is to keep them healthy with our limited resources. Although numerous groundbreaking achievements have been noticed in the health care sector for the recent years, the health care expense is still sky high and it has become an issue that even the developed countries are worry DOI : 10.5121/ijwmn.2014.6304 
about. According to the data provided by the Kaiser Family Foundation [4] the per capita expenditure of the health care is increasing at an exponential rate in some countries as shown in Figure 1. With such a high and continuously increasing healthcare expenses, medical care for the ageing people is becoming progressively challenging. One of the reasons for this high medical expense is hospitalization cost. The senior citizens are the most frequent visitors to the hospitals. They visit the hospitals for their medical treatment. Sometimes they have to stay there for a certain period of time for follow up of their medical treatment. Their staying in a hospital not only incurs expenses, but also incurs loss of patient's mobility. Remote Health Care Monitoring System (RHCMS) has been proposed as a solution to this problem. The main concept is to monitor a patient from a remote location and to provide her/him with necessary medical advices. The RHCMS has numerous advantages compared to conventional healthcare systems. Some of the advantages include (a) monitoring a patient, (b) responding to an emergency, (c) assisting patient mobility, (d) shortening hospital stay, and (e) reducing medical expenses. By using RHCMS the physical conditions of the patients can be monitored for twenty hours a day and seven days a week. The emergency services can be provided to the patients with a minimum delay. The patients can be served without going to a health care facility and admitting there. The healthcare professional can perform the follow up from a remote location and hence a patient needs to stay in a hospital for a short period of time. In a nutshell RHCMS reduces expenses related to the medical services.

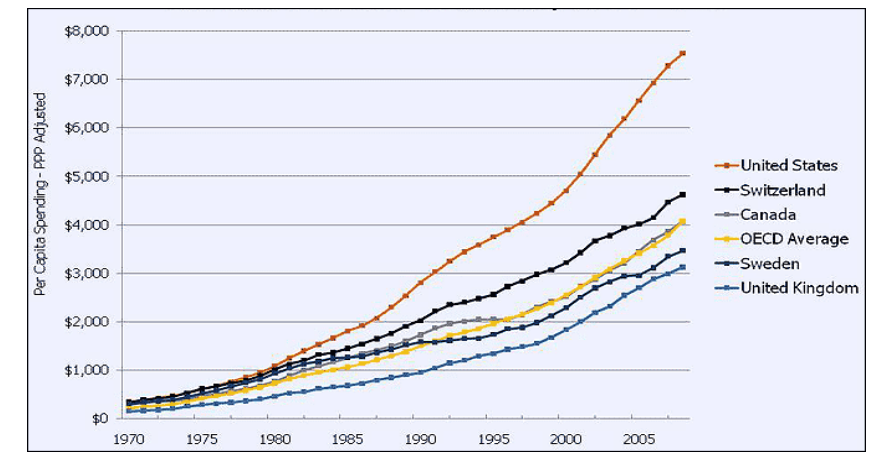

Figure 1 Per capita health acre expenditure of the World

Most of the proposed RHCMSs are based on wireless technology. The evolution of the wireless network has experienced a very fast-paced. Since the introduction of the IEEE 802.11 protocol wireless networks have experienced a huge market demand. Within the four years of the introduction wireless networks became very popular because of its portability, convenience, ease of installation, and low cost [5]. In that time period about 7.5 million households in the United States deployed wireless network. Wireless networks and medical sensors have been combined in RHCMS. There have been many medical sensors available in the market. Most of the sensors can measure and display critical health monitoring data such as the pulse rate, blood pressure, temperature, and blood sugar of a patient. One of the limitations of the proposed RHCMS is its limited coverage area. The measured data cannot be transmitted beyond a certain distance. Thus, it is not possible for the healthcare professionals to monitor the medical conditions of a patient from a distant location. In a hospital either the nurse or the physician has to move from one patient to another patient for monitoring them. Hence, it may not be possible for them to monitor a patient's health conditions all the time. This situation can be even worse when they have to take care of a large number of patients in a hospital at a given time. In order to overcome the above mentioned limitations an on-line health monitoring system has been proposed in this work. The 
proposed system can monitor the temperature, pulse, muscle, and ECG data of a patient. The proposed system has been designed by using the ZigBee technology. A major portion of this system has been implemented in LabView. Hence, the proposed system is reconfigurable as per users' need. The proposed system has been tested and verified in order to ensure its accuracy and reliability. The system consumes a very low power. The system consumes a very low power because it transmits signal only if the monitored parameters (i.e., temperature, heart beat rate etc.) go outside their normal ranges. Otherwise, the system puts the transmitter into a sleep mode to save energy. Hence the proposed system has a long operating life. The rest of the paper is organized as follows. Section 2 presents some related work. The system model of the proposed RHCMS has been presented in section 3. The implementation and results have been presented in section 4 . This paper is concluded with section 5 .

\section{RELATED WORKS}

Numerous prototypes for remote health care system can be found in the literatures. Since this work is based on the ZigBee technology, we focus only on the remote health care systems that have been designed based on the ZigBee technology.

One of the early works on health care monitoring system has been proposed in [6]. The proposed system is suitable for patients, senior citizens, and others who need continuous monitoring of their health. The proposed system can monitor the ECG signals of a patient based on Session Initiation Protocol (SIP) and a ZigBee network. The system consists of a wireless ECG sensor, ECG console, ZigBee module, SIP register, a proxy server, a database server, and wireless devices.

Simultaneous monitoring of the biomedical signals from multiple patients has been addressed in [7]. The proposed network is based on IEEE 802.15.4 standard and the ZigBee technology. The authors have proposed an optimized source routing protocol to control the network load. Some other issues including energy consumption, network lifetime, and delivery ratio have also been addressed in the same work.

An intelligent remote healthcare system based on power line communication and the ZigBee network has been proposed in [8]. The system consists of physiological sensors, a ZigBee/PLC gateway, and some special software. The physiological data are collected by the physiological sensor and are sent to a controlling center through a ZigBee/PLC gateway. The data are stored and analysed at the controlling center.

A low power microcontroller based patient bed monitoring system has been presented in [9]. Resistive bend sensor has been used for minimizing the harmful effects of bedsores, which is a common problem in hospital's intensive care units and assistive living environments during rehabilitations. The proposed system is able to replace the current wired system and it supports continuous patient monitoring by enabling the patient bed mobility.

A Wireless Body Area Sensor network (WBASN) based on the ZigBee technology has been presented in [10]. All nodes in the WBASN are connected as star topology and central node (i.e., access point) is used to control the network. The data collected by the access point is transferred to a hospital network over mobile communication network. The authors have proposed a novel "wake-up on-demand" mode of network operation. According to this mode the network "sleeps" when the most of the circuits are turned off to reduce power consumption. Once the WBASN is waked up all modules begin to work and all biomedical signals are obtained, stored, and transmitted.

Reliability of data transmission for healthcare monitoring system has been investigated in [11]. The authors have suggested that there is always a chance for loosing physiological data when a 
number of ZigBee devices operate in a hospital at a given time. Although the medium access control is taken care of by the MAC layer, the authors have designed and implemented a new medium access control algorithm to ensure reliable data transmission of the physiological data.

A telemedicine information monitoring system consists of vital sign monitoring devices, a healthcare gateway, and a health service information platform has been proposed in [12]. Among these components the healthcare gateway is the most critical component. The ZigBee module is used to transmit information between the vital sign monitoring devices and the healthcare gateway. The vital sign monitoring devices include ECG, SPO2, blood pressure, glucose, and body temperature. The data is then relayed to a healthcare service information platform. The system is based on Service Oriented Architecture (SOA) concept to provide the healthcare management for people who are suffering from chronic illness.

A remote patient monitoring system based on the ZigBee wireless sensor network and the Internet Things has been introduced in [13]. The system generates electronic medical records that are saved in a database. After analysing the data the proposed system can send feedback about the diagnosis, medical programs, and proposals to a remote location. The system uses the ZigBee network for real time transmission of the physical data. The data processing and information releasing have been implemented by a database program.

A wearable remote healthcare system for assessing hydration status and visceral fat accumulation by using Bioelectrical Impedance (BI) analysis has been proposed in [14]. The authors have designed a ZigBee based BI to replace the conventional wired BI. The proposed system consists of BI measurement circuit integrated with $0.35 \mu \mathrm{m}$ CMOS technology and a transducer circuit of the ZigBee module.

Two alternative systems have been proposed for the deployment of the ZigBee based wireless personal area network (WPANS) for remote patient monitoring in the general wards of a hospital in [15]. In the first approach a single WPAN is considered for gathering and transmitting physiological data from the patients in a ward. In the second approach multiple WPANS are considered. The simulation results show that the multiple WPANS out-perform the single one in respect of efficiency and reliability for data transmission.

An expandable wireless health monitoring system based on ZigBee has been proposed in [16]. The proposed system can monitor the temperature and pulses of a patient wirelessly. The test result presented therein shows that the proposed system can monitor the temperature and pulse of a patient with a high accuracy.

An ambient care system (ACS) framework to provide remote monitoring, emergency detection, activity logging, and personal notification services has been proposed in [17]. The proposed system consists of Crossbow MICAZ devices, sensors, and PDA enabled with ZigBee technology. The authors concluded that the combination of the ZigBee technology together with a service oriented architecture is the best option for ACS services.

A wireless sensor gateway (WSG) has been proposed in [18] to monitor patients' health. The main objective of this project is to monitor the cardiovascular status of a patient. Biological signals like ECG, pulse wave, and body weight are the important parameters for the cardiovascular monitoring of a patient. The proposed gateway is deigned to receive data from wireless sensors through a ZigBee interface and to forward the same to a personal computer via Bluetooth interface.

A ZigBee based system for remote monitoring of $\mathrm{SPO}_{2}$ has been proposed in [19]. The system consists of $\mathrm{SPO}_{2}$ sensor devices, a router, and Personal Area Network (PAN) co-ordinators. All 
the devices are based on MCU and ZigBee chip. The sensor devices measure $\mathrm{SPO}_{2}$ data from patients and transmit the data to the router. The router sets schedule for data transmission to each device by using a hierarchical routing. The proposed system also contains a web-based management system so that the patient data can be published in the web.

A real-time rehabilitation platform for patients and aged people has been proposed in [20]. The proposed system can collect data about the real-time walking acceleration of the patients. By analysing the gait sequences the computer based rehabilitation system can figure out the normal gait and abrupt falling of the people. The system also includes an ECG detector to monitor the health condition of the patients and the aged people.

A prototype of smart sniffing shoes has been designed for monitoring the foot health of a patient in [21]. The proposed system consists of chemical sensor array installed inside the shoe. The ZigBee technology has been used for the data communication. A technique called principal component analysis (PCA) has been used to monitor the foot health of a patient.

Another ZigBee based health monitoring system has been proposed to monitor temperature, heart rate, blood pressure, and movements of patients in [22]. The main component of the system is an electronic device worn on the wrist or finger of a high risk patient. The system uses a number of sensors including an impact sensor to detect the fall of a person. The system can monitor a medically distressed person and send an alarm to a caretaker system connected to a remote computer.

A similar work for monitoring the patient's pulse has been presented in [23]. The proposed system can monitor the pulses of a patient from a remote location and it can also administer necessary medical treatment. The proposed system consists of a pulse sensor, ZigBee module, and ATmega218P microcontroller. The pulse measured by the sensor is sent to a coordinator through a ZigBee interface. The test results show that the proposed system can cover up to 30 meter distance.

A prototype model for cardiovascular activity and fitness monitoring system based on IEEE 11073 family has been proposed in [24]. The IEEE 11073-10441 defines the set of protocols for tele-health environment at application layer and the rest of the communication is taken care of by the medical grade ZigBee network. The test results show that the proposed system can report severe cardiovascular malfunctioning without compromising the mobility.

A remote heart sound and lung sound monitoring system has been proposed in [25]. The authors have solved the problem related to simultaneous transmission of heart and lung sounds. Sensors have been used to collect the heart and lung sounds and then FastICA is used to separate these two signals. The sound signals are then sent to a remote location via internet for diagnosis.

A low cost sleep monitoring system based on polysomnography has been proposed in [26]. The authors have introduced some innovative sensor pillow and bed sheet system that employ the ZigBee wireless network. To monitor the respiration of the patient a sensor array of force sensitive resistors (FSR) based on polymer thick film device has been used. The sensor array is able to classify and verify the respiratory rate during sleep.

A portable ECG monitoring system has been proposed in [27]. The proposed system is controlled by MSP430 single chip computer, which amplifies and filters the patient's ECG signals and sends data to a central controller using a ZigBee wireless transmission module. Another similar prototype of a ZigBee based ECG signal monitoring system has been proposed in [28]. A PC 
based GUI interface has been developed to provide ECG signal processing task and health care video tracking and management functions.

In this work we have proposed a remote health monitoring system based on the ZigBee technology and LabView software. The proposed system can monitor ECG signals, muscle power, temperature, and heartbeat of a patient from a remote location. In contrast to other related works we used National Instruments' LabView software for implementing the project. The LabView constitutes a graphical programming environment that can acquire data (i.e., biomedical signals). The LabView relies on graphical symbols rather than textual language to describe programming actions. The principle of dataflow governs program execution in a straightforward manner. We chose LabView software because it is easy to program and it has powerful data acquisition system. In addition the output data generated by the LabView program can be easily acquired into hardware. The data acquisition is performed by using Data Acquisition System (DAQ) provided by the National Instrument. The use of DAQ reduces the complexities of the circuits. Since the major portion of this work is implemented in LabView, the proposed system can be easily reconfigured and adapted to accommodate more options in future. The system can send data to a remote location for diagnosis. The system can also publish data in the internet so that the concerned healthcare professionals can monitor their patients from anywhere around the World at any time.

\section{SYSTEM MODEL}

The proposed system consists of a set of biomedical sensors attached with the body of a patient. A wireless transmitter is used to send the data to a wireless receiver connected to a local monitoring unit. In this work we used six biomedical sensors to monitor heart beat rate, temperature, changes in muscles power, and ECG signals of a patient. These sensors convert the physiological changes of the patient's body into biomedical signals. The conditioning circuit (i.e., Arduino microcontroller) reads the data from the sensors and controls the transmission of data to a monitoring unit. The monitoring unit displays the data that is used by the physicians for necessary medical advices. The wireless receiver consists of Xbee that receives data and sends it to the local monitoring unit. The monitoring unit can display, record, and analyze the data. It can send reports as well as alarming messages to the healthcare professionals. The system block diagram of the proposed system is shown in Figure 2.

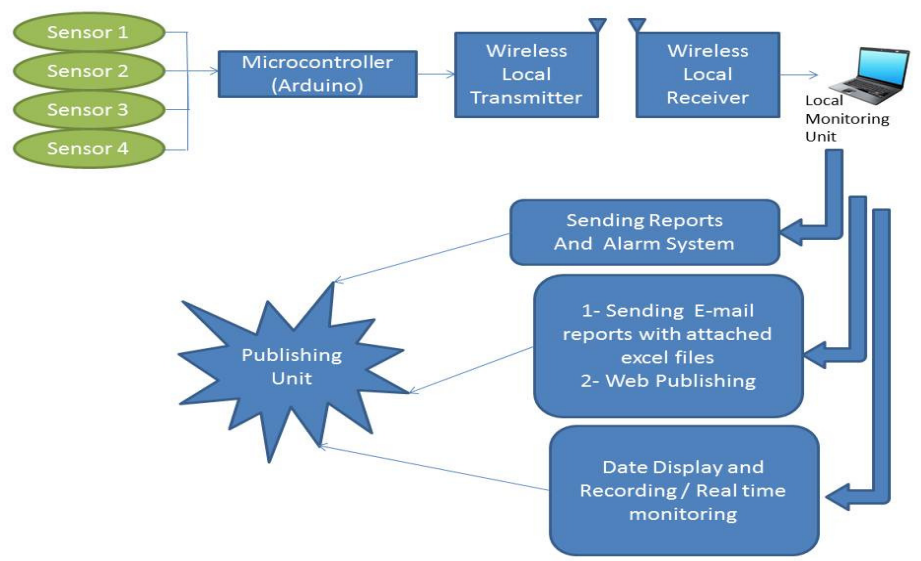

Figure 2 The system block diagram. 
Based on the customer requirements the system hardware can be easily modified to accommodate more sensors. The data transmission can also take place via wired or wireless channels. The proposed system can be connected to the Internet for global communication. In addition, the proposed system is carefully implemented in hardware and software system so that it can be adapted to fulfill the user's requirements. Since accuracy is one of the most important issues in biomedical signal processing, the proposed system has been field tested extensively to ensure its accuracy. The system can continuously monitor the health of a patient twenty four hours a day. The proposed system is also able to inform the healthcare professionals about any unusual health conditions of a patient. The doctors can also use the publishing system incorporated with the system. When the measured data exceeds the allowable normal range, the system can send an alarm message to the concerned healthcare professionals. The system can facilitate healthcare professionals to perform immediate medical diagnosis and to administer the medical treatment if needed. The system measures different physical parameters of a patient by using four different sensors as shown in Figure 3. The microcontroller receives the signals from the sensors and processes them before sending them to a ZigBee transmitter module. The transmitter module transmits the signal that is received by the receiving antenna of the ZigBee receiver.

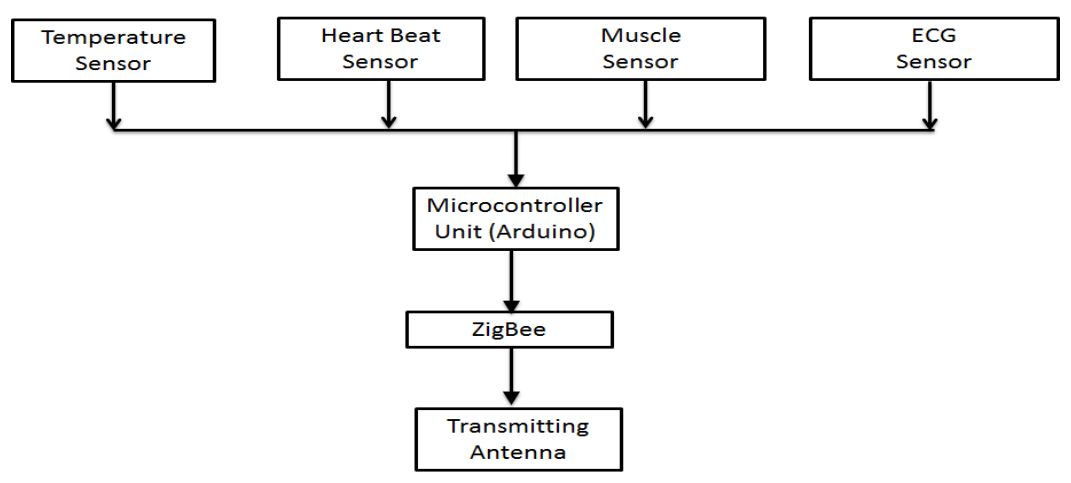

Figure 3 System block diagram of transmitter section

The system block diagram for the receiver is shown in Figure 4. The receiver antenna receives the data sent by the transmitting antenna and then the data are sent to a PC (i.e., Monitoring Unit) for display. The Monitoring Unit sends report using the internet to the concerned healthcare professionals.

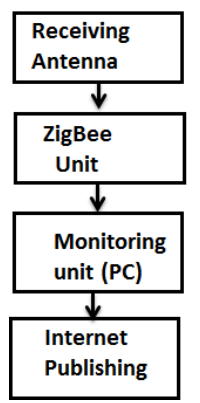

Figure 4 System block diagram receiver section

The temperature sensor used in our system is shown in Figure 5(a) and the associated program flowchart in shown in Figure 5(b). We used LM35 sensor for our project. The LM35 is a high 


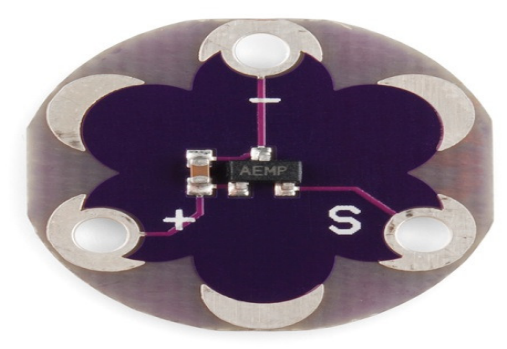

(a) Temperature Sensor

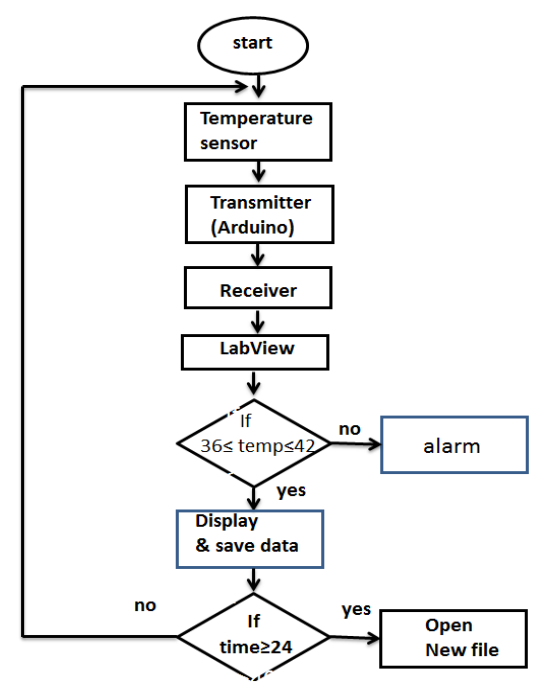

(b) Flowchart

Figure 5 The temperature measurement.

precision integrated temperature sensor. It generates an analog voltage depending on the temperature of the patient's body. The sensor output voltage is linearly proportional to the body temperature. The sensor circuitry is sealed and is not subject to oxidation. The LM35 generates a higher output voltage than thermocouples. The sensor can measure temperature and generate signal that is sent to a microcontroller. The data are then transmitted by the ZigBee to the PC. The sensors are connected to the I/O port of the PIC microcontroller (i.e., Arduino). The output voltage is converted into temperature by a simple conversion factor. As shown in Figure 6 the temperature sensor measures the temperature and converts it into electrical signal. The electrical signal is then processed by a microcontroller and the LabView software. Finally, it is displayed in the monitoring unit. We set the normal body temperature of a patient in the range of $36^{\circ} \mathrm{C}-40^{\circ} \mathrm{C}$. If the temperature reading is less than $36^{\circ} \mathrm{C}$ or more than $40^{\circ} \mathrm{C}$ degree the alarm will be $\mathrm{ON}$ and it will send an alert message to the concerned healthcare professional.

The heart beat sensor used to measure the heartbeat of the patient is shown in Figure 6(a). This sensor monitors the flow of blood through a clip that is attached with a fingertip. The sensor has a laser that emits light through the skin and measures the reflection of the laser due to the flow of the blood. The heart beat rate of an individual may vary. At rest, an adult man has an average pulse rate of 72 beats per minute. Athletes normally have a lower pulse rate compared to that of a less active people. On the other hand children have a higher pulse rate (approx. 90 beats per minute). We set the critical pulse rate at 120 beats per minute. The flowchart for the heart pulse 
sensor is shown in Figure 6(b). The sensor measures the heart beats and converts them into electrical signals. Then the receiver antenna sends the data to the Monitoring Unit for displaying the data. If the pulse rate is less than 120 beats per minute, the alarm will be $\mathrm{ON}$ and it will alert the concerned authority.

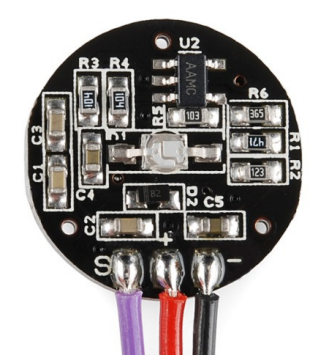

(a) Heart beat sensor

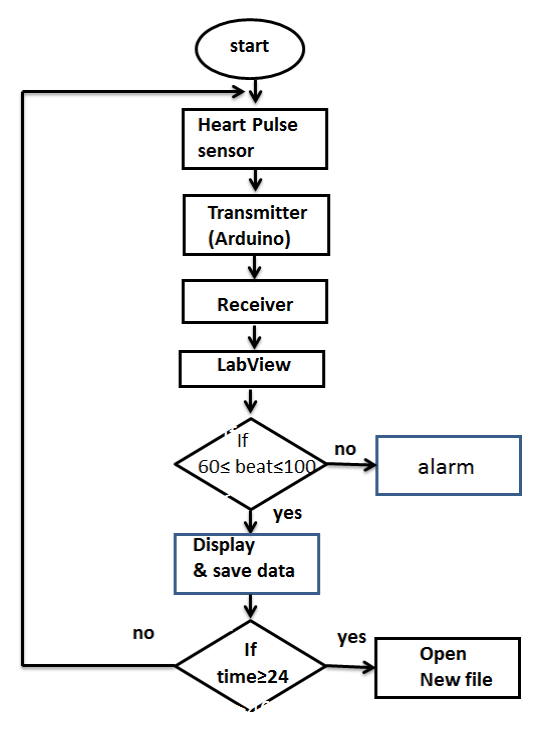

(b) Flowchart

Figure 6 The measurement of heart beat rates

The muscles sensor used in this project is shown in Figure 7(a). The sensor detects the changes in the muscle force and converts it into variable resistive readable values. The local monitoring unit coverts the resistive date from the muscle sensor to a power signal. The main purpose of the muscle sensor is to measure the power from the muscles. In some cases elderly people who are unable to move or disabled and their muscles power decreases over a time period, the sensor sends an alert message to health care service provider. If the patient is moving or standing still or sleeping, the status of the patient's movement can also be monitored by our proposed system. The flowchart of the muscle sensor is shown in Figure 7(b). The transmitter sends the signal which is received by the receiver. The receiver sends the data to the Monitoring Unit for graphical display. If the reading is less than 150 or more than 500 the alarm will be $\mathrm{ON}$ and it will alert the health care service provider. 

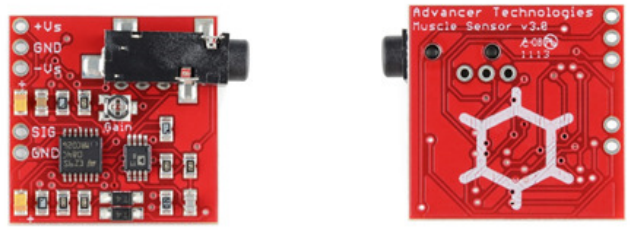

(a) Muscle sensors

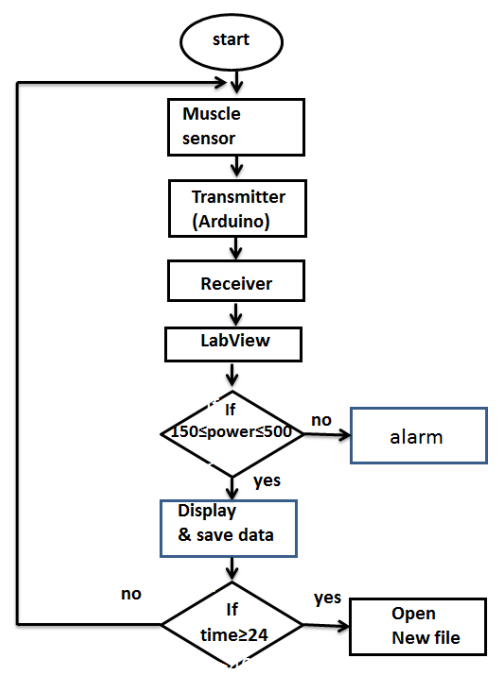

(b) Flowchart

Figure 7 The measurement of muscle power

ECG is an important biomedical parameter and is used clinically in diagnosing various diseases and conditions of a patient's heart. The acquisition of a real time ECG signal requires an expensive CARDIART machine and only experienced cardiologist can interpret the ECG signal. However, in developing and under developed countries people cannot make use of this facility because they live in remote areas. In this proposed system we developed an accurate, low cost, and user friendly real time ECG acquisition system for monitoring general cardiac abnormalities. By using the proposed system the ECG data can be monitored and analysed from a remote location via Web browser. Hence, the system supports long distance diagnosis. The system can generate a report of the patient's condition by using the ECG data. The hardware ECG is mainly consisting of an electronic circuit, which uses amplifiers and switches to amplify the readings from the sensor so that it can be read and displayed. The software ECG monitors the readings from the sensors and then converts them into the readings based on a defined formula. The reading of the software ECG has been verified with that of a CARDIART machine in order to ensure reliability and accuracy. The proposed system measures ECG data from four sensors placed at four different places of the patient's body as shown in Figure 8 and provides an output as shown in Figure 9. The detail operation of the ECG is illustrated in Figure 10. First, the microcontroller (i.e., Arduino) takes the signals from the sensors and converts them into readable values using some defined formula. Then the data are transferred from the transmitter to the receiver (i.e.,Xbee). Then the LabView program combines these readings from the four sensors and provides one variable output. The ECG signal flow diagram is shown in Figure 10. 
International Journal of Wireless \& Mobile Networks (IJWMN) Vol. 6, No. 3, June 2014
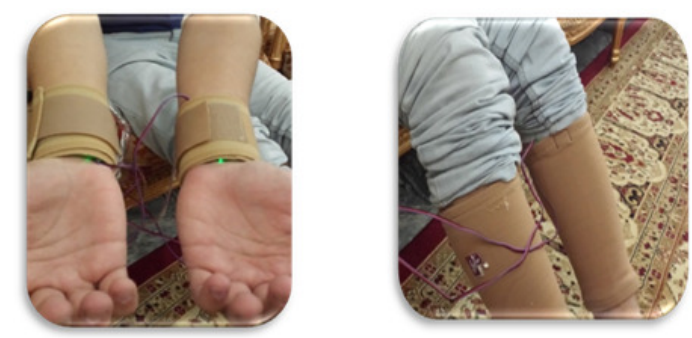

Figure 8 ECG Sensors

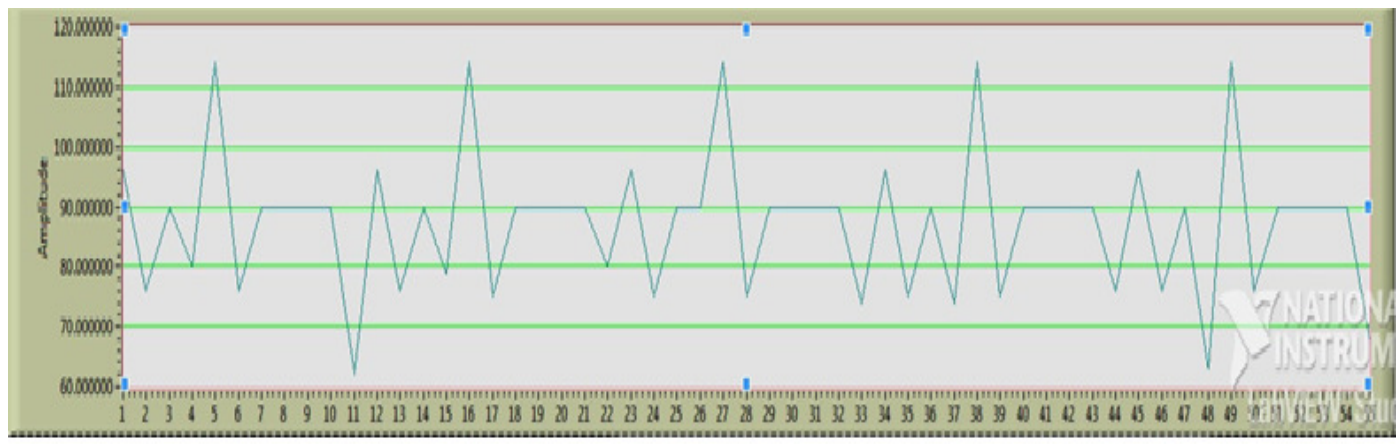

Figure 9 The output of ECG sensors.

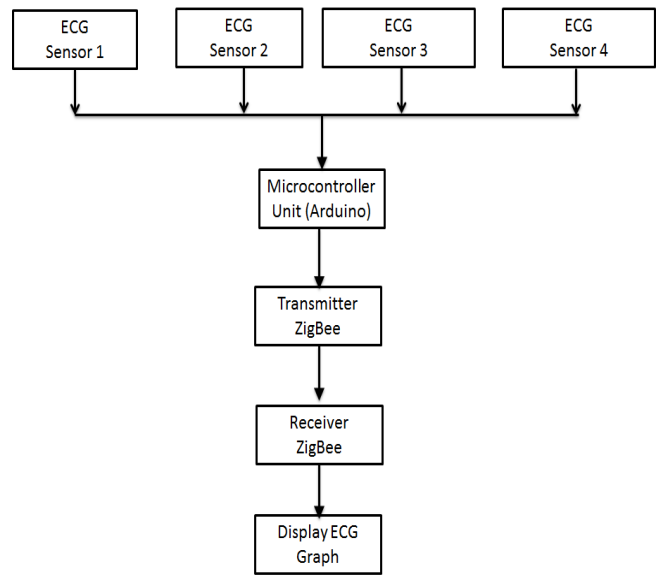

Figure 10 ECG signal flow diagram

\section{IMPLEMENTATION AND RESULTS}

The proposed system provides a patient with continuous health monitoring service. The signals generated by the sensors are processed by a built-in microcontroller. The processed data are then transmitted by ZigBee wireless transmitter. Finally the received data is sent to a PC. The proposed system works as follows: (a) the user will wear the sensors, (b) the sensors will start 


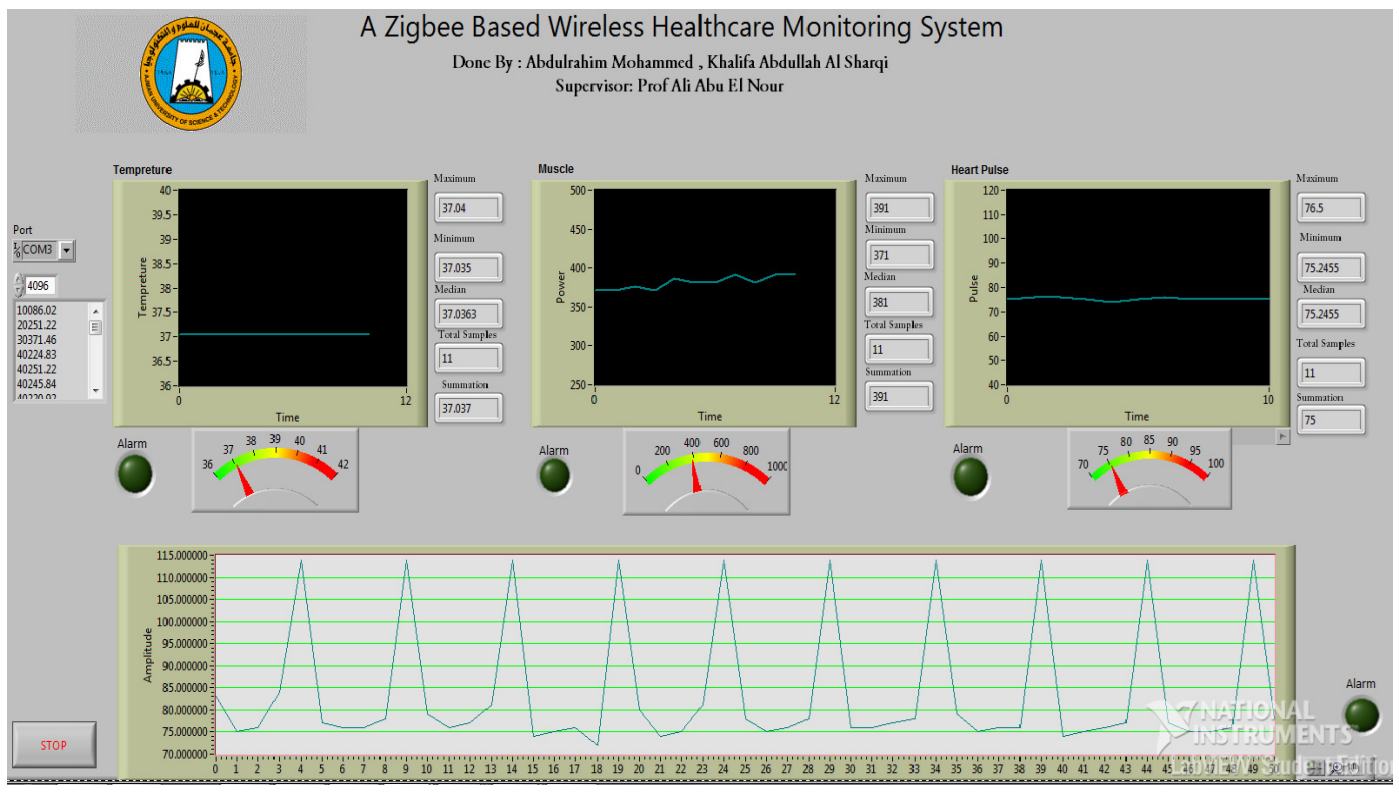

Figure 11 Labview front panel

reading the temperature, heart beats rate, muscle power, and ECG data, and (c) the program will send the monitored data wirelessly and interfaces between the LabView software and the hardware. The front panel of the proposed system is shown in Figure 11.

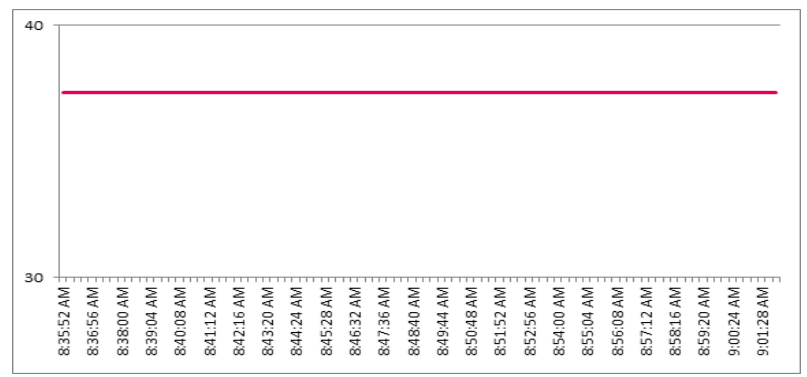

Figure 12 Output of the temperature sensor

Figure 12 shows a sample of the temperature sensor data. It shows the variation of the temperature with respect to time. It is depicted in this figure that the temperature is in the normal range from 8:35 AM to 9:01 AM which means that the patient's body temperature is within the normal range. Since the body temperature is within the set normal range, the system will not send any alarm message to the healthcare professional.

Figure 13 shows a sample output of the heart pulse sensor. The output shows that there are the changes in the heart pulse rate from time to time. The figure illustrates that the patient heart pulse rate varies between 75 beats per minute and 76 beats per minute for the monitored period of time. Since the heart beat rate is within the normal limit, we assume that the patient is at stand still condition. 


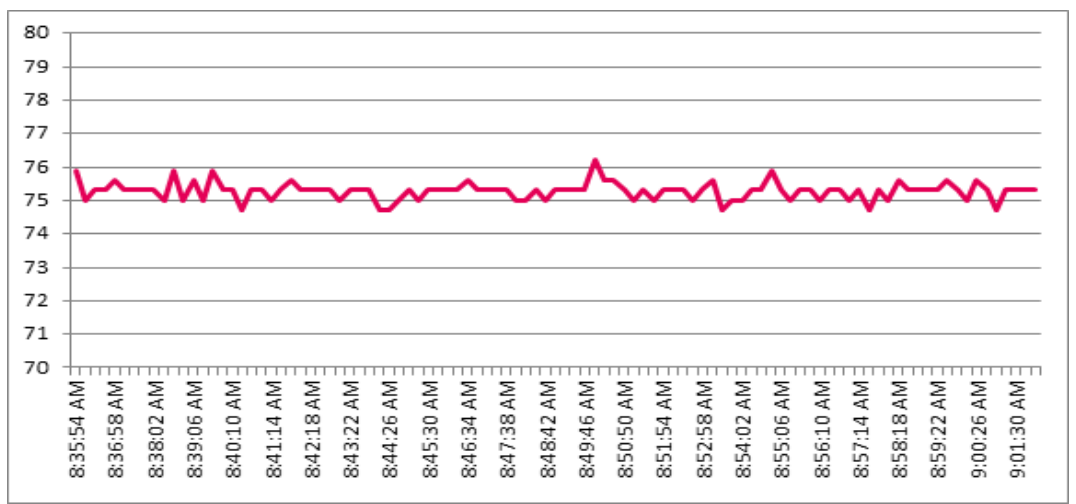

Figure 13 The output of heart pulse sensor

Figure 14 shows a sample of the output of the muscle sensor attached with the arm. It shows the percentage usage of the patients' muscles $(\mathrm{Max}=1000)$ with respect to time. The figure shows the percentage usage of the patients' muscles is varying between 200 and 310 for the monitored period of time (i.e., from 8:40 AM to 9:01 AM). This variation indicates that the patient is moving and hence the muscles power is increasing with respect to time.

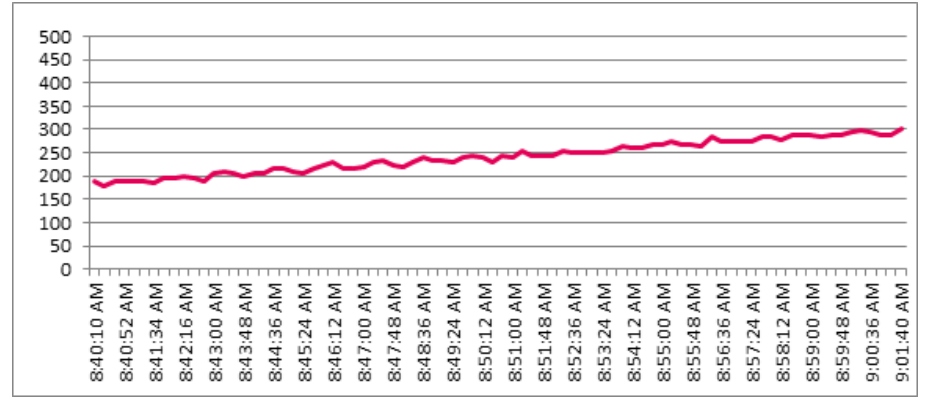

Figure 14 The output of the muscle sensor (Arm)

\section{Conclusions}

A reliable wireless healthcare monitoring system has been designed and successfully implemented in this work. The proposed system has been field tested. The test results show that the proposed system is able to monitor the body temperature, heart pulse rate, ECG signal, and muscle power with enough accuracy. Since the proposed system is based on ZigBee, we can conclude that it is a low power and low cost system. Moreover, major part of the proposed system has been implemented in using LabView software. Hence, the proposed system is easily reconfigurable and it can be connected to the Internet easily. The system is also able to store physiological data of patients for 24 hours a day and seven days a week. In future the proposed system can be extended to include more sensors that can measure more parameters like diabetes and blood pressure. The proposed system is flexible enough to include such kind of modifications. 
International Journal of Wireless \& Mobile Networks (IJWMN) Vol. 6, No. 3, June 2014

\section{REFERENCES}

[1] Linking Population, Poverty, and Development available at www.unfpa.org.

[2] Health United States, 2004 available at http://www.cdc.gov.

[3] Projected Population of the United States, by age and sex:2000 to 2050 available at http://www.census.gov

[4] Medical Costs available at kff.org/health-costs

[5] Home Digital Home: Wi-Fi Gets a Place At The Table available at http://www.wirelessfidelitymag.com/

[6] Bonam Kim, Youngjon Kim, InSung Lee, and Ilsum You, “ Design and Implementation of a Ubiquitous ECG Monitoring System Using SIP and the ZigBee Networks”, In the proceedings of the Future Generation Communication and Networking (FGCN 2007), December 6-8, 2007, Jeju, Korea, pp. 599-604

[7] Fariborz H., Moghawemi, M., and Mehrkanoon, S, “ The design of an intelligent wireless sensor network for ubiquitous healthcare", In the Proceedings of the International Conference on Intelligent and Advanced System, November 25-28, 2007, Kualalumpur, Malaysia, pp. 414-417

[8] Xi Xueliang, Tao Cheng, and Fang Xingyuan, " A health care System based on PLC and ZiGbee", In proceedings of the International Conference on Wireless Communication, Networking and Mobile Computing, September 21-25, 2007, Shanghai, China, pp. 3063-3066

[9] Manohar, A. and Bhatia, D., “ Pressure Detection and Wireless Interfaces for patient bed, " In the Proceedings of the IEEE Biomedical Circuits and Systems Conference, November 20-22, , 2007, Baltimore, MD, pp. 389-392

[10] Xiao Hu, Jianging Wang, Qun Yu, and Waixi Liu, “ A Wireless Sensor Network Based on ZiGbee for Telemedicine Monitoring System", In the Proceedings of the $2^{\text {nd }}$ International Conference on Bioinformatics and Biomedical Engineering, May 16-18, 2008, Shanghai, China, pp. 1367-1370.

[11] Juang J.Y., and Lee, J.W., “ ZigBee Device Access Control and Reliable Data Transmission in ZigBee Based Health Monitoring System", In the Proceedings of the $10^{\text {th }}$ International Conference on Advanced Communication Technology, February, Guagwan-Do, 2008, pp. 795-797

[12] Hsu Chih-Jen, “ Telemedicine information monitoring system”, In the Proceedings of the $10^{\text {th }}$ International Conference on E-health Networking, Application, and Service, Julu 7-9, 2008, Singapore, pp. 48-50.

[13] Jingram Luo, Yulu Chen, Kai Tang, Juwen Luo, “ Remote monitoring information system and its applications based on the Internet Things", In the proceedings of the International Conference on Future Biomedical Information Engineering, December 13-14, 2009, Sanya, pp. 482-485

[14] Ramos, J., Austin, J.L., Torelli, G., Duque-Carnillo, J.F.,” A wireless sensor network fort fat and hydration monitoring by bioimpedance analysis," In the Proceedings of the $6^{\text {th }}$ International Wearble Micro and Nano Technologies for Personalized Helath, June 25-26, 2009, Oslo, pp. 4952

[15] Yuanlong Liu, and Sahandi, R.," ZigBee network for remote patient monitoring on general hospital wards", In the Proceedings of the XXII International Symposium on Information, Communication, and Automation technologies, October 29-31, 2009, Bosnia, pp. 1-7

[16] Chengcheng Ding, Xiaopei Wu, Zhao Lu, “ Design and Implementation of the ZigBee-based Body Sensor Network System", In the Proceedings of the $5^{\text {th }}$ International Wireless Communciations, Networking, and Mobile Computing, September 24-26, 2009, Beijing, pp. 1-4.

[17] Martin, H., Bernades, A.M., Bergesio, L., and Tarrio, P., " Analysis of key aspects to manage wireless sensor networks in ambient assisted living Environments", In the proceedings of the $2^{\text {nd }}$ International Symposium on Applied Sciences in Biomedical and Communciation Technologies, November 24-27, 2009, Brastislava, pp.1-8

[18] Becher, K., Fugueredo, C.P., Muhle, C., and Ruff, R., “ Design and realization of a wireless sensor gateway for health monitoring", In the proceedings of the Annual IEEE Conference on Engineering in Medicine and Biology Society, August 31-Sept 4, 2010, Buenes Aires, pp. 374-377

[19] Zhou Yan, and Zhu Jiaxing, “Design and implementation of ZiBee based wireless sensor network for remote $\mathrm{SPO}_{2}$ monitor", In the proceedings of the International Conference on Future Computer and Communication, May 21-24, 2010 Wuhan, pp. V2 278-V2 281 
[20] Tian Lan, and Xiaogiong Li, " Gait Analysis via a high resolution triaxial accelartion sensor based on ZigBee Technology", In the proceedings of the International Conference on Complex Medical Technology, May 25-28, Beijing, 2013, pp. 697-702

[21] Seesaard T., Lorwongtragol, P., and Nilpanapan, T, “ An smart sniffing shoes based on embroided sensor array", In the proceedings of the $10^{\text {th }}$ International Conference on Electrical Engineering/Electronics, Computer, Telecommunication, and Information Tehcnology, May 1517, 2007, Krabi, pp. 1-4

[22] Deppa, A., and Kumar P.N., " Patient health monitoring based on ZigBee Module", In the proceedings of the International Conference on Optical Imaging Sensor and Secuirty, July 2-3, 2013, Coimbatore, pp. 1-4

[23] Niswar, M., Ilham, A.A., Palantei, E, and Sadjad, R.S., “ Performance evaluation of a ZigBee based Wireless sensor network for monitoring patient's pulse status", In The proceedings of the International Conference on Information technology and Electrical Engineering, Yogyakarta, Octiber 7-8, 2012

[24] Gangwar, D.S., "Biomedical sensor network for cardiovascular fitness and activity monitoring" In the proceedings of the IEEE Point of Care Healthcare Technolonogies (PAT), January 16-18, 2013, Bangalore, pp. 279-282

[25] Yi Zhang, Sixuan Chen, Jin Wu, Yuan Luo, " Remote heart and lung sound monitoring system design based on ZigBee", In the proceedings of the $6^{\text {th }}$ International Conference on Biomedical Engineering and Informatics, October 16-18, 2012 Chongqing, pp. 1109-1111. s

[26] Lokavee, S., Puntheeranurak, T., Kerdeharoen, T., Wahanwisuth , W.," Sensor pillow and bed sheet system: Unconstrained monitoring of respiration rate and posture movements during sleep", In the Proceedings of the IEEE Conference on Systems, Man, and Cybernetics, October 14-17, Seoul, 2012, pp.

[27] Hongli Yang and Jhing Chai, " A portable wireless ECG monitoring system based on MSPCBOFGA39" In the proceedings of the International Conference on Intelligent Computation and Biomedical Instrumentation, December 14-17, 2011, Wuhan, pp. 148-151

[28] Hui-Yang H Sia, Linag-Hung Wang, Feng-chi Lin, and Chien-Chou Chen, “ Design and implementation of a wireless ECG acquisition and Communication system with health care services", In the proceedings of the International Symposium on Bioelectronics and Bioinformatics, November 3-5, Suzhou, 2011, pp. 25-28 